\title{
La Translocación in vitro Citoplasma/Núcleo del Factor de Transcripción Embrionario OCT-4 en Células Perivasculares Propone a la Aorta Como un Nicho Quiescente de Células Madres Adultas
}

\author{
In vitro Translocation Cytoplasm/nucleus of Embryonic Transcription Factor OCT-4 \\ in Perivascular Cells Suggests that Aorta as a Niche of Quiescent Adult Stem Cells
}

Jesús Herrera-Bravo*; Enrique Montiel-Eulefi*; Talita Glaser**;

Marcelo Garcés $^{* * *}$; Pamela Leal ${ }^{* * * *}$ \& Alexander Henning Ulrich ${ }^{* *}$

HERRERA-BRAVO, J.; MONTIEL-EULEFI, E.; GLASER, T.; GARCÉS, M; LEAL, P. \& HENNING, U. A. La Translocación In Vitro Citoplasma/Núcleo del Factor de Transcripción Embrionario OCT-4 en Células Perivasculares Propone a la Arteria Aorta Como un Nicho Quiescente de Células Madre Adultas. Int. J. Morphol., 31(4):1430-1438, 2013.

RESUMEN: Las células perivasculares tienen un origen común en las células madre embrionarias y en los vasos sanguíneos que proporcionan un nicho para la mantención de su troncalidad. La expresión de marcadores embrionarios y de células indiferenciadas, como también la gran variedad de fenotipos celulares generados desde los pericitos, podrían ser explicados por la capacidad de estas células de ser inducidas a un estado "stemness" cuando son tratadas con factores adecuados. Nuestros resultados describen la expresión de células con OCT-4 citoplasmático en una ubicación anatómica perivascular donde de su nicho se encuentra en la región intima de la aorta en rata. In vitro las células aisladas por el método de explante que promueve el aislamiento de células migratorias desde los tejidos muestran un fenotipo con un citoplasma alargado y que expresan aSMA, PDGFRa y b, siendo estos dos últimos marcadores específicos de pericitos. En estas células se presenta una tranlocación a la variante nuclear de OCT-4 que ha sido descrito como el principal regulador de los procesos de autorrenovación y pluripotencia. La expresión de OCT-4 confirma y amplía aún más las observaciones obtenidas en nuestras investigaciones anteriores y demuestra que células madre se encuentran en los vasos sanguíneos en un microambiente que, probablemente, les permite que sobrevivan y permanezcan en reposo como un tipo de célula troncal quiescente.

PALABRAS CLAVE: Troncalidad; Pericitos; Translocación; OCT-4; Aorta de rata.

\section{INTRODUCCIÓN}

Nuestro grupo de investigación, ha identificado que las células perivasculares tienen un origen común en las células madre embrionarias y los vasos sanguíneos proporcionan un nicho para la mantención de su troncalidad (MontielEulefi et al., 2009). De esta manera, las células aisladas de los vasos poseen actividad pluripotente y con capacidad de diferenciarse en los distintos tipos celulares provenientes de las tres capas germinativas. Estas células perivasculares presentan un fenotipo de pericitos expresando los marcadores especificos "Thymocyte differentiation antigen 1" (Thy1/CD90), "alpha-smooth muscle actin" (aSMA), "Plateletderived growth factor receptor a" (PDGFRa) (Montiel-Eulefi et al., 2012; Tárnok et al., 2010).
En la actualidad, la idea que todas las células madre mesenquimales son pericitos es un tema en discusión debido a que pueden expresar también marcadores encontrados en este tipo celular (Caplan, 2008; da Silva Meirelles et al., 2008; Dore-Duffy \& Cleary, 2011). Los pericitos pueden ser inducidos a un estado embrionario caracterizado por la expresión de "Stage-specific mouse embryonic antigen" (SSEA-1), un antígeno específico presente en células madre embrionarias y a progenitores neuronales, a través del uso de protocolos definidos (Dore-Duffy et al., 2006; Montiel-Eulefi et al., 2012), incluso se ha descrito que los pericitos pueden diferenciarse a osteoblastos, condroblastos, fibroblastos, adipocitos y células del músculo liso (Canfield et al., 1996; Korn et al., 2002; Reilly

\footnotetext{
* Centro de Biotecnología en Reproducción (CEBIOR-CEGIN-BIOREN). Depto, de Ciencias Básicas. Fac. de Medicina, Universidad de La Frontera,Temuco. Chile. ** Departamento de Bioquímica, Instituto de Química, Universidade de São Paulo, São Paulo, Brasil

**** Center of Plant, Soil Interaction, and Natural Resources Biotechnology. (BIOREN). Universidad de La Frontera, Temuco. Chile.

${ }^{* * * * *}$ Laboratory of Molecular Pathology, Department of Pathology, Faculty of Medicine, CEGIN-BIOREN, Universidad de La Frontera, Temuco. Chile.
} 
et al., 1998; Terskikh et al., 2001). Sin embargo, la expresión de marcadores embrionarios y de células indiferenciadas, como también la gran variedad de fenotipos celulares generados desde los pericitos, podrían ser explicados por la capacidad de estas células de ser inducidas a un estado "stemness" cuando son tratadas con factores adecuados.

El estado "stemness" ha sido definido por varios autores como la capacidad de un grupo celular de mantener un estado indiferenciado, proliferante y con la capacidad de diferenciarse a cualquier fenotipo celular procedente de los tres linajes embrionarios. Este grupo celular, que se encuentran principalmente en estados tempranos del desarrollo embrionario, son capaces de activar la expresión genética de ciertos factores vinculados con el estado pluripotente y reprimir la expresión de otros vinculados con la diferenciación. Yamanaka \& Takahashi (2006) fueron premiados por el descubrimiento de cuatro factores vinculados con la reprogramación de fibroblastos adultos en células madre pluripotentes embrionarias (SOX-2, cMYC, KLF-4 y OCT4). De los cuatro factores, OCT-4 ha sido descrito como el actor principal en el mantenimiento de la pluripotencia y la autorrenovación de las células madre embrionarias. Durante la diferenciación de las células del macizo interno del blastocisto, OCT-4 disminuye su expresión dando origen a la expresión de otros genes vinculados con el desarrollo del ser vivo, por cuanto la presencia de este factor de transcripción en tejido adulto: médula ósea, cordón umbilical, epidermis, corazón, páncreas, testículos y epitelio bronquial (Ratajczak et al., 2007), sugiere, sin ser conocida su ubicación y características de este tipo celular, que son algún tipo de célula madre pluripotente se mantiene en la adultez.

Muchos trabajos actuales han identificado células madre basando sus resultados en la expresión de marcadores de "stemness" tales como OCT-4, Sox-2 o Nanog. Esta investigación se basa en OCT-4, el principal factor de transcripción vinculado con el estado stemness y en humanos esta localizado en el cromosoma 6 y puede codificar para dos variantes OCT-4A, que se encuentra principalmente en el núcleo, regulando los procesos de autorrenovación y pluripotencia y OCT$4 \mathrm{~B}$, que estando localizado en el citoplasma y su función no se vincula con el estado "stemness" (Lee et al., 2006). La expresión de estos marcadores en células madre adultas hace que sea tentador pensar en este tipo celular como objeto de trabajo en terapia celular, sin embargo, la expresión de OCT4A o B como parámetros de pluripotencia para la designación de una células madre adulta debe ser considerado cuidadosamente (Landazuri \& Taylor, 2005).

Estudios actuales indican que en las paredes de las arterias existen distintos tipos celulares con características de progenitores celulares (Zengin et al., 2006), consistente con los resultados anteriores, científicos de la Universidad de Bologna, Italia, mostraron que en partes torácicas de la aorta, procedentes de donantes humanos sanos, existen dos distintas subpoblaciones con marcadores de células madre, células CD34+ y células c-Kit+ (Pasquinelli et al., 2007). Sin embargo, el presente trabajo ha demostrado la presencia de OCT-4 en células periciticas ubicadas en un nicho perivascular de la parte torácica de la aorta y sugiere a este fenotipo celular como fuente de células madre adultas tal y como se ha señalado en observaciones previas (Crisan et al., 2008; Pasquinelli et al., 2010). Desde ahí, estas células podrían migran y dependiendo de los factores del medio, diferenciarse a distintos fenotipos celulares, manteniendo de esta forma la reparación tisular en los organismos adultos.

\section{MATERIAL Y MÉTODO}

\section{Modelos de estudio}

Animales. Rata Sprague Dawley (Rattus norvegicus) provienentes del Bioterio de la Universidad de la Frontera, Temuco.

Líneas celulares. Las células perivasculares, utilizadas en este trabajo, han provenido del cultivo primario de células procedentes de explantes de aorta de rata Sprague Dawley obtenidas como se describió previamente por Montiel-Eulefi et al. (2012). Como control fueron utilizadas células endoteliales de vena umbilical humana (HuVEC) Donadas por C. Herrera (investigador del Departamento de Ciencias Preclínicas de la Facultad de Medicina de la Universidad de La Frontera). La línea de células madre embrionarias, E14TG2a fue donado por el Dr. Alexander Henning Ulrich, del Instituto de Química. Universidade de São Paulo. Los ensayos moleculares, se realizaron en el laboratorio de Biomedicina del Centro de Biología de la Reproducción (CEBIOR), el cultivo celular, la Citometría de Flujo y Citometría Confocal se realizaron en Scientific and Technological Bioresource Nucleus (BIOREN) pertenecientes a la Universidad de La Frontera.

Cultivo celular. Cultivo primario. La aorta de rata Sprague Dowley fue disecada adaptando el protocolo propuesto por Howson et al. (2005). Posterior a la disgregación mecánica sobre la placa de cultivo las células procedentes del vaso, comenzaron a migrar y luego de 24 horas fueron adhiriéndose a la placa en medio de cultivo.

Cultivo líneas de células. Las células de aorta de rata y HuVEC fueron cultivadas en placas de cultivo para tejido y células Orange Scientific (Bélgica). Las dimensiones de las 
HERRERA-BRAVO, J.; MONTIEL-EULEFI, E.; GLASER, T.; GARCÉS, M; LEAL, P. \& HENNING, U. A. La Translocación In Vitro Citoplasma/Núcleo del Factor de Transcripción Embrionario OCT-4 en Células Perivasculares Propone a la Arteria Aorta Como un Nicho Quiescente de Células Madre Adultas. Int. J. Morphol., 31(4):1430-1438, 2013.

placas utilizadas dependen del tipo de ensayo a realizar. Las células fueron cultivadas en placas de de $60 \mathrm{~mm}$ en Medio DMEM alto en glucosa (Invitrogen), suplementado con piruvato de sodio $(0,11 \mathrm{~g} / \mathrm{l})$, bicarbonato de sodio $(3,7 \mathrm{~g} / \mathrm{l})$, penicilina-estreptomicina (100 unidades/ml) y $10 \%$ FBS (GIBCO) a $37^{\circ} \mathrm{C}$ y $5 \% \mathrm{CO} 2$. Las células fueron plaquedas cada 5 días hasta que alcanzaron el $80 \%$ de confluencia. Luego las células fueron disgregadas con tripsina/EDTA 0,5\% y cultivadas nuevamente para que pudiesen proliferar. Para conservar las células, estas fueron congeladas en nitrógeno líquido $\left(-197^{\circ} \mathrm{C}\right)$ hasta su posterior uso. Las células embrionarias se cultivan independiente de células alimentadoras, en placas recubiertas de gelatina $(0,2 \%)$ con DMEM con alta glucosa suplementado con suero fetal de bovino (FBS al 15\%), piruvato sódico [1mM], aminoácidos no esenciales MEM (1\%), factor inhibidor de la leucemia murina $\left[1 \times 10^{3} \mathrm{U} / \mathrm{ml}\right]$, $\beta$ mercaptoetanol $[0,1 \mathrm{mM}]$, penicilina $[50 \mathrm{U} / \mathrm{ml}], y$ estreptomicina $\left[50 \mathrm{mg} / \mathrm{ml}\right.$ a $37^{\circ} \mathrm{C}$ con $\mathrm{CO}_{2}(5 \%)$. Las células se subcultivaron cada 2-3 días hasta que se alcanza el $80 \%$ de confluencia para desarrollar la experimentación.

\section{Inmunofluorescencia indirecta para Oct4}

In vitro. Células en cultivo fueron cultivadas $24 \mathrm{~h}$ en placas Fluordish TM con medio de proliferación y se fijaron con paraformaldehído [4\%] en PBS durante la noche a $4{ }^{\circ} \mathrm{C}$, se lavaron con PBS y se permeabilizaron con Triton X-100 [0,05\%] durante 5 minutos y se incubaron con solución de bloqueo con $5 \%$ leche desnatada, incubadas con rabbit antiOCT-4 con una dilución de 1:200 durante toda la noche a 4 ${ }^{\circ} \mathrm{C}$ y una hora con el anticuerpo secundario Alexa fluor 633. Los núcleos se marcaron con DAPI.

In situ. Por medio de una disecación se extrajo la partetorácica de la aorta y fue fijada en formalina al 3-4\% para posterior estudio histológico. Los tejidos se deshidrataron en una batería de alcoholes (de 70\% a 100\%), y toluenos a distintas concentraciones, luego se incluyeron en parafina y se tallaron en tacos para realizar los cortes histológicos. Se realizaron los cortes de 5 micras con un micrótomo Leitz. Los cortes se pegaron en portaobjetos tratados con poli-L-Lisina $(0,1 \%)$, se desparafinaron, y rehidrataron con alcoholes de $100 \%$ a $70 \%$. Una vez en agua destilada, se lavaron con PBS, se permeabilizaron con Triton $\mathrm{X}-100$ [0,05\%] durante 5 minutos y se incubaron con solución de bloqueo con $5 \%$ de leche desnatada. La inmunohistoquímica se realizó incubando el tejido con anticuerpo anti-OCT-4 de conejo con una dilución de 1:200 durante toda la noche a $4^{\circ} \mathrm{C}$ y una hora con el anticuerpo secundario Alexa fluor 633 de cabra anti conejo. Los núcleos se marcaron con DAPI. Se cubrieron con glicerol y se observaron en Microscopio Laser Confocal Olympus Fluoview 1000, del BIOREN de la Universidad de La Frontera, Chile.

\section{RT-PCR and PCR}

RT-PCR. El ARN total se extrajo de las células con Trizol (Invitrogen) y se examinó tanto para la calidad y la cantidad con un NanoDrop ${ }^{\circledR}$ ND-1000 (Thermo Fisher Scientific). La transcripción inversa (RT) se realizó con Oligo-dT y $1 \mathrm{mg}$ de ARN tratado con ADNasa y transcriptasa inversa M-MLV (Promega). Las reacciones sin la enzima se llevaron a cabo como control. Para cada muestra, una décima parte del ADNc de la reacción de RT se utilizó como templado para la PCR. Las condiciones de reacción fueron, $37,5 \mathrm{mM}$ de $\mathrm{KCl}, 1,25 \mathrm{mM}$ de 2-5-trifosfato de desoxinucleótido, $25 \mathrm{mM} \mathrm{MgCl}, 1 \mathrm{U} \mathrm{ADN}$ polimerasa GoTaqFlexi (Promega), y 1mM cada uno de los cebadores sentido y reverso en un volumen de $20 \mathrm{ml}$. La PCR se realizó a $94^{\circ} \mathrm{C}$ durante $5 \mathrm{~min}$, seguido por 30 ciclos de $94^{\circ} \mathrm{C}$ durante $35 \mathrm{~s}, 55^{\circ} \mathrm{C}$ durante $30 \mathrm{~s}$, y $72^{\circ} \mathrm{C}$ durante $30 \mathrm{~s}$. Los cebadores para las reacciones de PCR fueron: aSMA cebador Sent: TGCTGGACTCTGGAGATG, Rev: GTGATCACCTGCCCATC (Amplicon 292pb); GAPDH Sent: CCTCTGGAAAGCTGTGGCGT; Rev: TTGGAGGCCATG TAGGCCAT (Amplicon 450pb); PDGFRa Sent: TCTCGGCATGACGGATTCTT, Rev: CCACACTGAAGGTTCCGTTGA (Amplicon 164pb), PDGFRb: Sent: GATTCCATGCCGAGTGACAGA, Rev: TCCCCAATGGTGGTTTTGC (Amplicon 149pb) (Howson, et al., 2005). Los productos de PCR se separaron mediante la realización de electroforesis en geles de agarosa que contienen bromuro de etidio y se visualizaron bajo luz UV.

Citometría de flujo. Las células fueron despegadas agregando 500ul de solución tripsina/EDTA (1x) por 5 min a $37^{\circ} \mathrm{C}$ y luego disgregadas mecánicamente haciendo pasar las células varias veces por una jeringa de $5 \mathrm{ml}$. La citometría de flujo (FCM) para la cuantificación de células Thy-1/CD90+ se realizó mediante la inmunotinción con un anticuerpo policlonal de conejo de la siguiente manera. Las células derivadas de explantes aórticos adultos se aislaron y se lavaron con PBS/EDTA [5 mM]. Las células se contaron y se diluyeron a 1.000 .000 células/ml en cada tubo. Las células se precipitaron por centrifugación a $1.000 \mathrm{~g}$, se permeabilizaron con PBS/Triton X-100 [0,5\%] y se bloquearon durante $1 \mathrm{~h}$ con $3 \%$ de BSA y IgG de conejo no específicos a temperatura ambiente. Anticuerpo de conejo anti-Thy-1/CD90 se añadió a la suspensión de células y se incubó a $4^{\circ} \mathrm{C}$ durante la noche en PBS que contenía $3 \%$ de BSA y $0,1 \%$ de Triton X-100, mientras que una población de células en ausencia del anticuerpo primario se utilizó como control. Las células se lavaron dos veces mediante PBS que contenía $0,3 \%$ de BSA y $0,1 \%$ de Triton X-100 seguido de una incubación de 30 min a temperatura ambiente con anticuerpo de cabra conjugado con Alexa 633 anti-IgG de conejo. 
El mismo procedimiento se realizó con células perivaculares y el anticuerpo de conejo anti OCT-4 y reveladas con el anticuerpo de cabra conjugado con Alexa 633 anti-IgG de conejo.

Las células se lavaron co PBS y se separaron en una única suspensión de células con un filtro de 80 micrómetros y se analizaron mediante un citómetro de flujo BD FACS Canto II (Becton y Dickinson). El posterior análisis de datos se realizó con el software Cyflogic 1.2.1 (2008).

Análisis estadístico. La prueba de t de Student se utilizó para determinar las diferencias de OCT-4 nuclear, siendo el valor de $\mathrm{p}<0,05$ considerado como significativo. El tratamiento estadístico se realizó con el software GraphPad Prism (GraphPad Software, Inc., San Diego, CA) para PC.

\section{RESULTADOS}

Las células perivasculares de aorta de rata expresan el marcador de células madre OCT-4 al igual que las células madre embrionarias
Las células fueron evaluadas por inmunofluorescencia indirecta contra OCT-4, a través, de microscopía confocal y mostraron expresión endógena al igual que células madre embrionarias que fueron usadas como control positivo (Fig. 1A, B y C). La línea celular HuVEC, que provienen del endotelio de las venas del cordón umbilical humano, no expresaran OCT-4 en cultivo y fueron utilizadas como control negativo (Fig. 1D, E y F). Los resultados muestran que las células obtenidas desde explante de aorta en cultivo expresan endógenamente este factor de transcripción principalmente en el núcleo (Fig. aG, H e I). El control de la técnica sin anticuerpo primario demuestra que esta marcación es específica para OCT-4 en las células perivasculares (Fig. aJ,K y L). La expresión de OCT-4 es confirmada mediante citometría de flujo indirecta, demostrando que aproximadamente el $70 \%$ de las células cultivadas expresan este factor de trascripción (Fig. 1M).

Los resultados confirman el estado inmaduro de las células periciticas al expresar el factor de transcripción OCT4, quien ha sido considerado como el actor principal de los mecanismos de autorenovación y pluripotencia. Por otro lado, siendo OCT-4 expresado en células madre embrionarias, la presencia de este factor de transcripción en células adultas

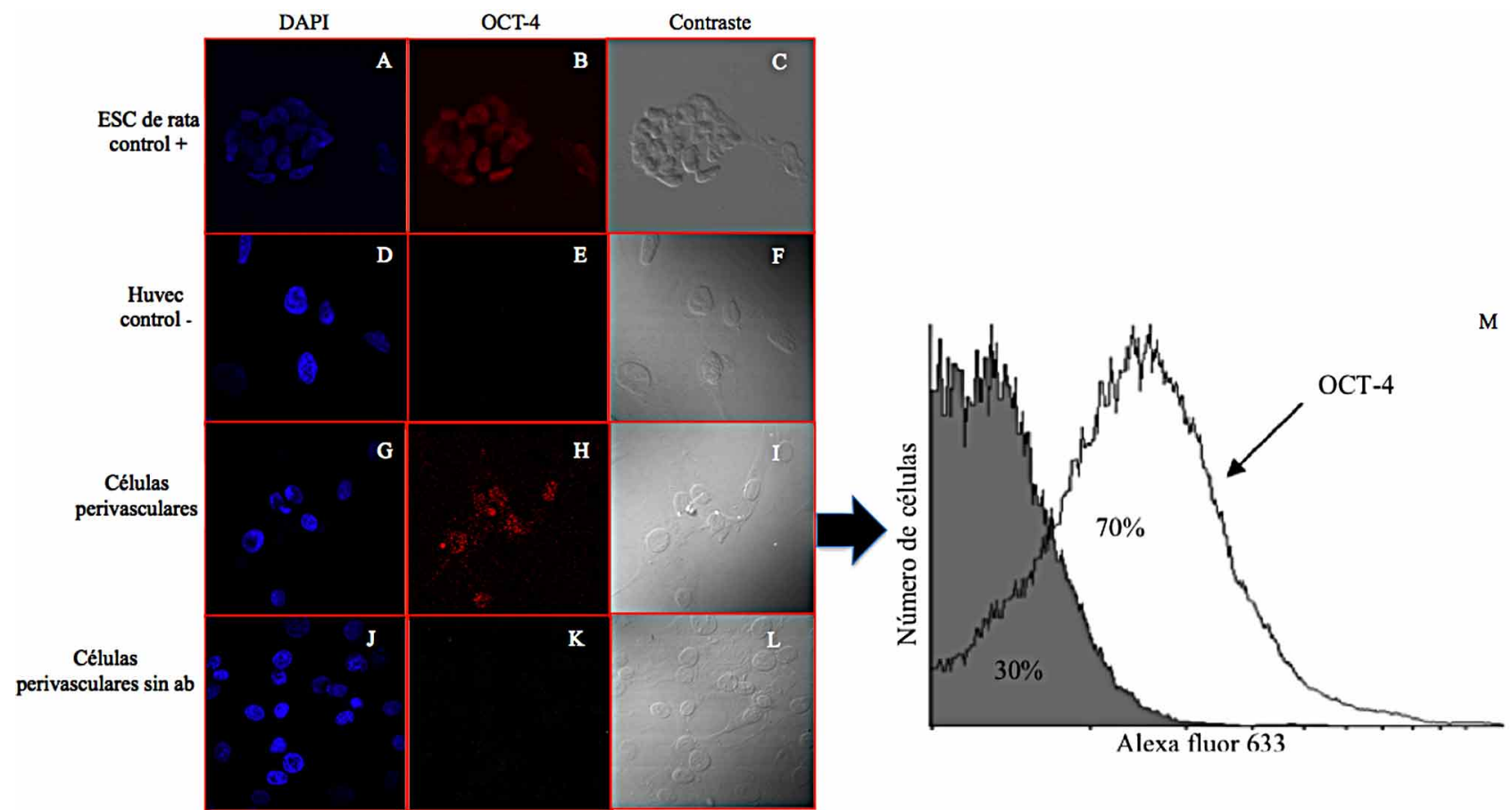

Fig. 1. Inmunofluorescencia indirecta para OCT-4 en células madre embrionarias y perivasculares. Las células fueron cultivadas 24 h en placas Fluordish TM con medio de proliferación, bloqueadas con 5\% leche, incubadas con anticuerpo hecho en conejo anti-OCT-4 y como anticuerpo secundario IgG de cabra anti conejo conjugado con Alexa fluor 633. Células madre embrionarias (A, B y C). Células del endotelio de las venas del cordón umbilical humano (D, E y F). Células perivasculares desde explantes de aorta de rata (G, H e I). Células perivasculares desde explantes de aorta de rata sin anticuerpo primario (J, K y L). Azul: DAPI. Rojo: Alexa fluor 633. (M) Citometria de flujo para OCT-4. Histograma señala que cerca del 70\% de la población celular expresa OCT-4 (flecha) y que un 30\% de la población no expresa el factor de transcripción (Curva gris). 

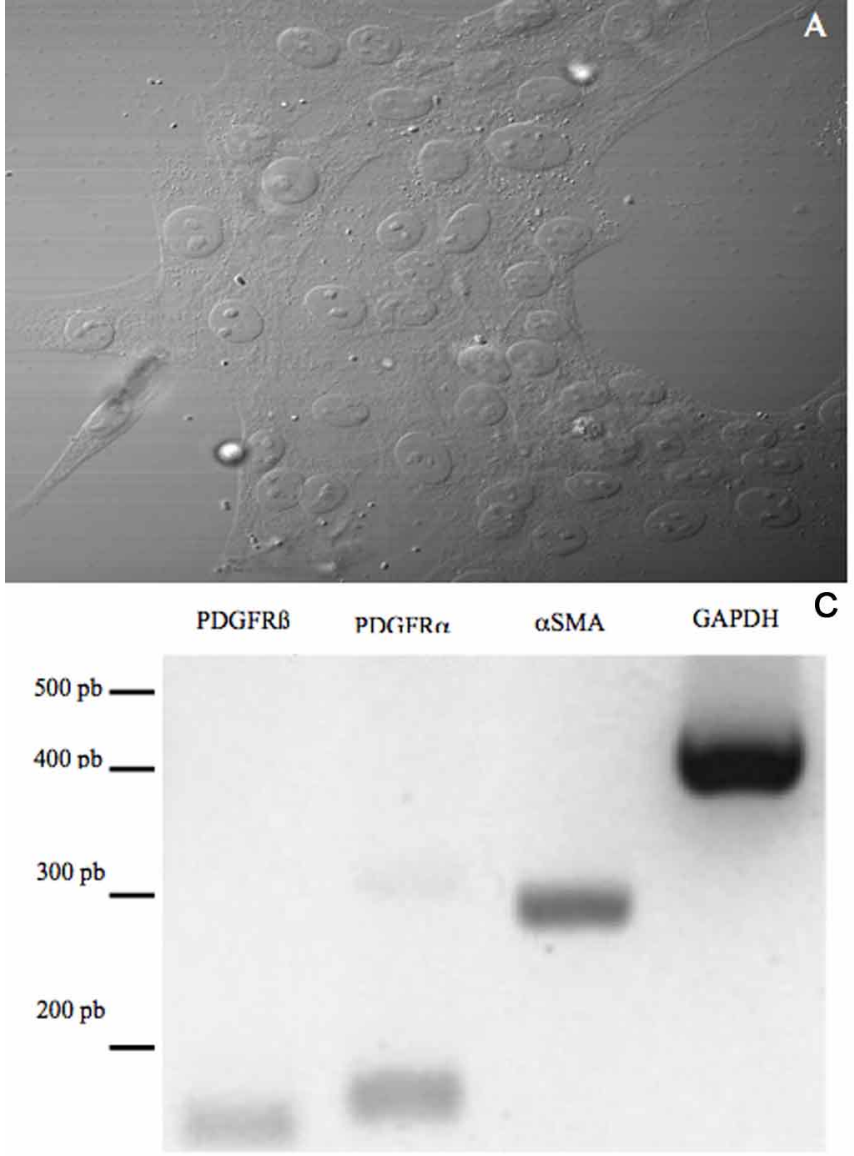
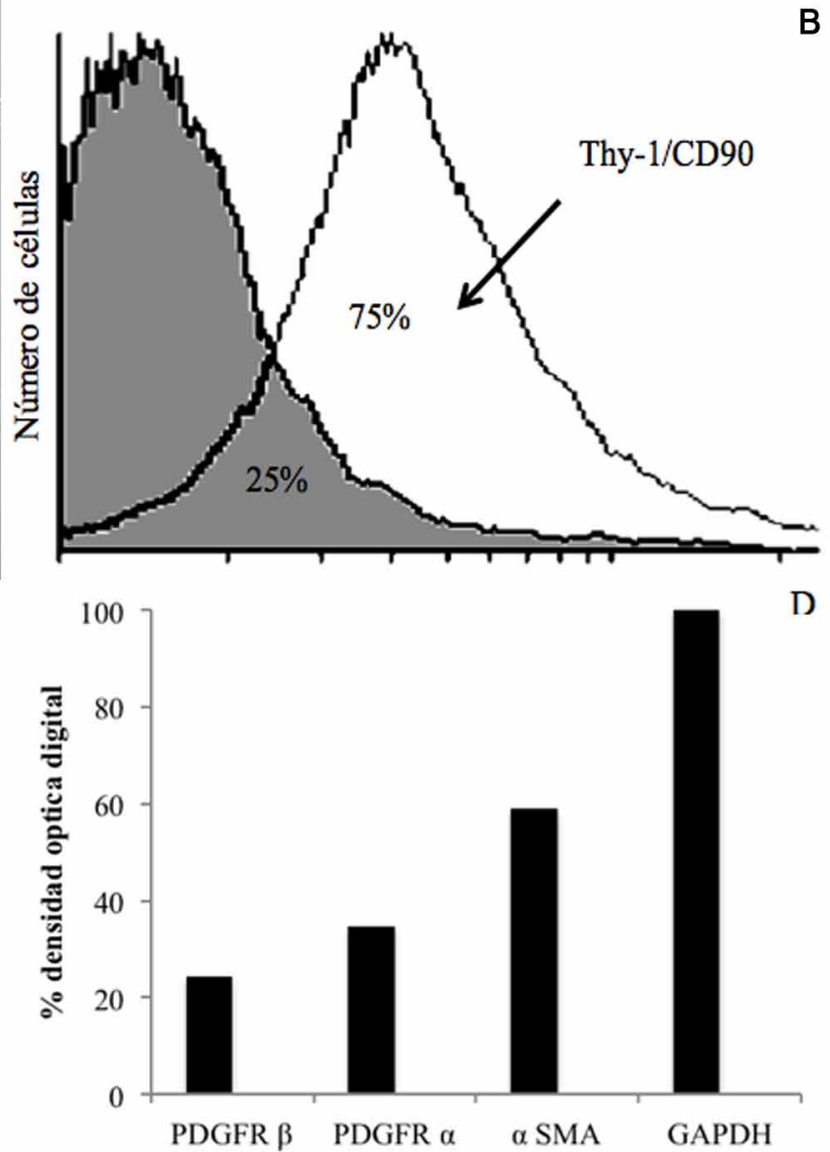

Fig. 2. Caracterización del fenotipo de pericito de las células perivasculares. Las células perivasculares de aorta presentan un fenotipo mesenquimal cuando son cultivadas en medio DMEM 10\% FBS (A). Histograma obtenido por citometría de flujo del marcador de pericito y células madre mesenquimales indica que el 75\% de la población celular expresa Thy-1/CD90 (flecha) y un 25\% no expresa el marcador (curva gris) (B). La PCR muestra que células perivasculares obtenidas desde explantes de aorta de rata adulta expresan marcadores de pericitos PDGFRa y b (C). GAPDH fue utilizado como control de la expresión. La cuantificación de la densidad óptica de los amplicones de cada gen es graficado respecto de la expresión de GAPDH (D).

sugiere nuevas evidencias al paradigma que señala que las células madre adultas tienen un origen perivascular.

\section{Caracterización de las células perivasculares/pericitos} en cultivo. Las células perivasculares procedentes del la aorta de rata Sprague Dawley, luego de 24 horas in vitro comenzaron a disgregarse y migrar, adhiriéndose a la placa de cultivo presentando fenotipo mesenquimal, con forma plana, un citoplasma extendido y estrellado, varios nucléolos, mostrando fibras de estrés a lo largo de su estructura (Fig. 2A). Las células perivasculares en cultivo fueron procesadas para citometría de flujo indirecta. Las células conjugadas con anticuerpo de conejo anti Thy-1/ CD90, un marcador descrito para células mesenquimales y periciticas, muestran que la población celular se encuentra enriquecida en células que expresan Thy-1/CD90 (Fig. 2B). La presencia de la expresión de genes previamente señala- dos como marcadores de células periciticas PDGFRa, aSMA, PDFGRb fue evidenciado por RT-PCR en células cultivadas bajo las mismas condiciones anteriores (Fig. 2C y D).

Inmuno detección in situ de OCT-4 en Aorta de rata adulta y su translocación in vitro. La microscopía confocal muestra que las células de la capa intima que rodean los vasos sanguíneos de la parte torácica de la aorta expresan OCT-4 (Fig. 3A y B) en una localización mayormente citoplasmática (Fig. 3C y D). Las mismas células en cultivo muestran una distribución distinta de OCT-4, principalmente nuclear (Fig. 4D, E y F). La comparación de la fluorescencia nuclear entre las células pericíticas de arteria con las mismas en cultivo (Fig.a $4 \mathrm{G})$, indica que existe una diferencia estadísticamente con en la presencia del factor de transcripción en el nucleo las células periciticas en cultivo $(\mathrm{P}<0,001)$. 


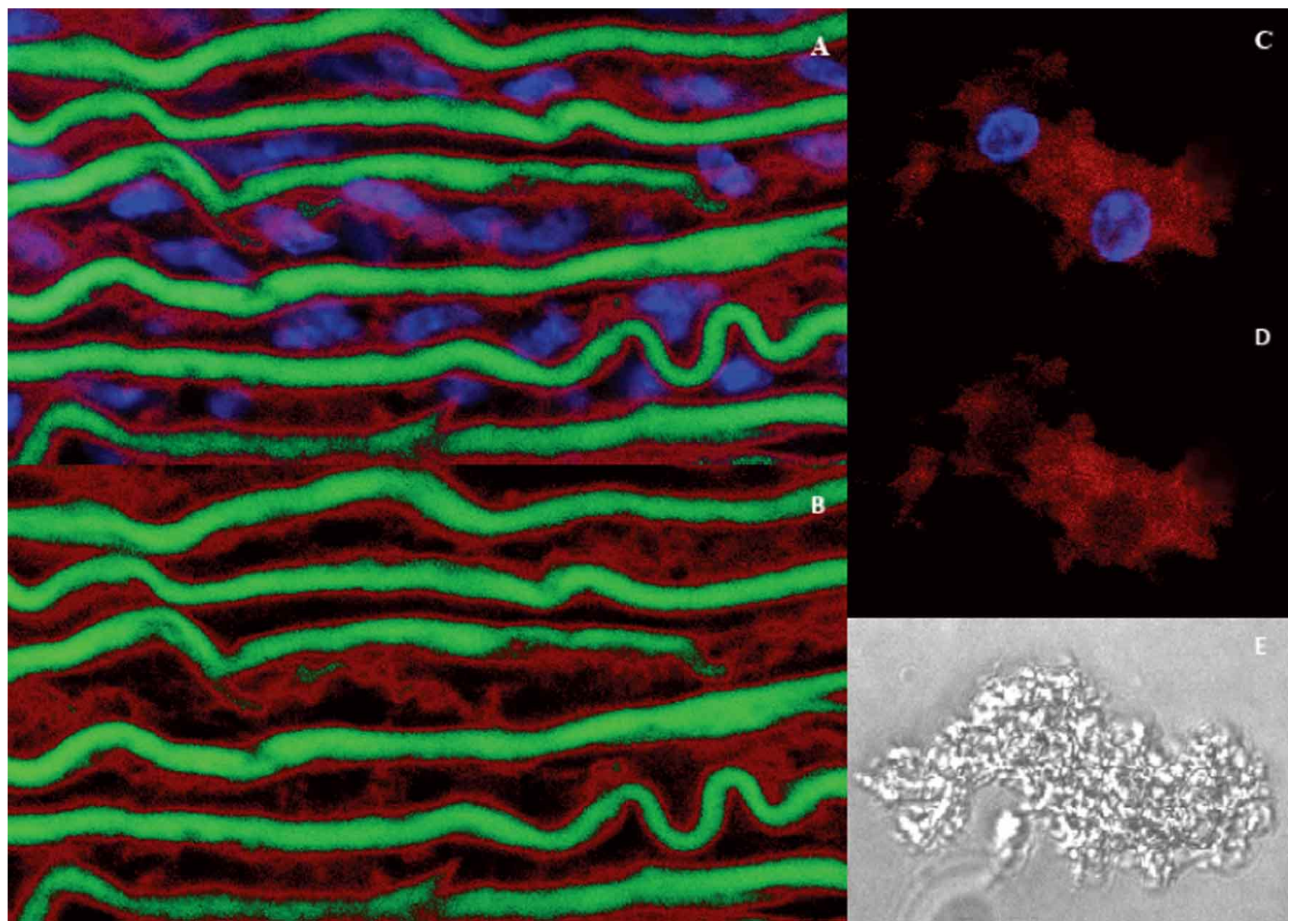

Fig. 3. Inmuno detección de OCT-4 en aorta de rata adulta. Corte longitudinal de parte torácica de la aorta de rata adulta. Autofluorescencia de Elastina (verde) se encuentra rodeada de células perivasculares que expresan OCT-4 endógeno (rojo) (A y B). Células perivasculares asiladas desde la aorta expresan OCT-4 (rojo) encontrándose en el citoplasma (C y D). Microfotografía confocal de contraste de fase de las células aisladas desde aorta (E). Los núcleos han sido marcados en azul con DAPI.
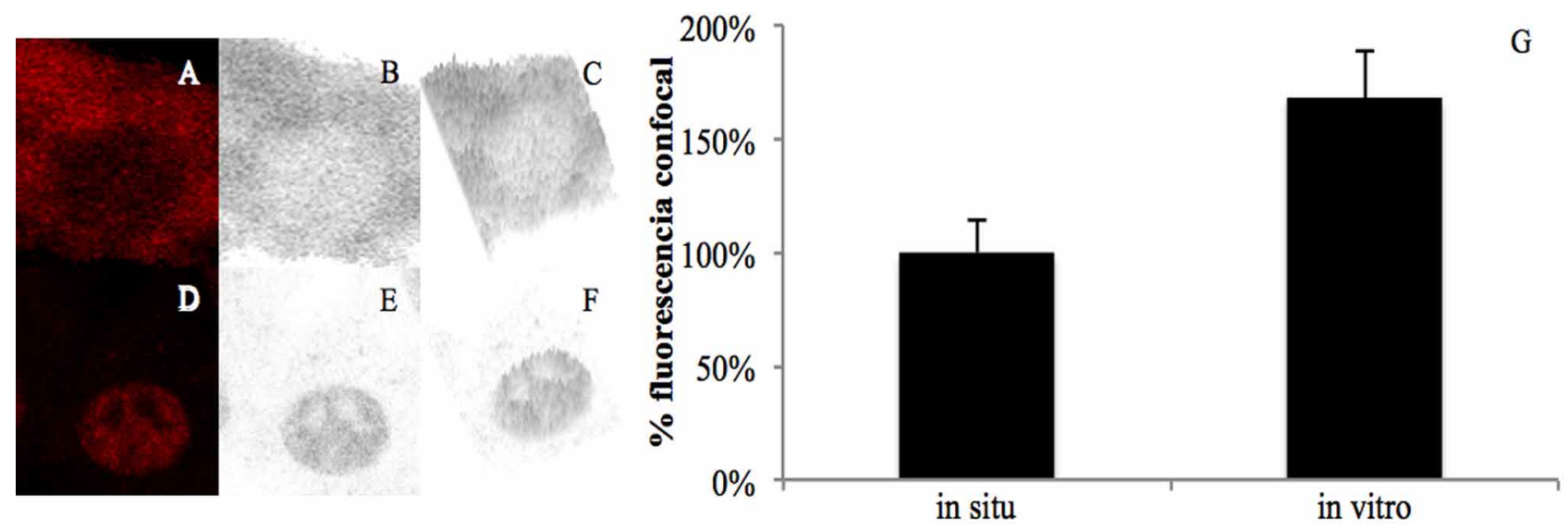

Fig. 4. Distribución nuclear de OCT-4 en células perivasculares de aorta de rata adulta en cultivo. La expresión nuclear de OCT-4 fue evaluado en células perivasculares in situ de tejido de aorta de rata adulta (A, B y C) y en células cultivadas desde explantes de aorta de rata adulta in vitro (D, E y F). Comparación de la expresión nuclear de OCT-4 en células perivasculares in situ de tejido de aorta y en cultivo in vitro $(\mathrm{G})$. El eje Y representa la intensidad de la fluorescencia de OCT-4 nuclear. La comparación se realizó a través de análisis de $t$ de Student con $\mathrm{p}<0,001$. 
HERRERA-BRAVO, J.; MONTIEL-EULEFI, E.; GLASER, T.; GARCÉS, M; LEAL, P. \& HENNING, U. A. La Translocación In Vitro Citoplasma/Núcleo del Factor de Transcripción Embrionario OCT-4 en Células Perivasculares Propone a la Arteria Aorta Como un Nicho Quiescente de Células Madre Adultas. Int. J. Morphol., 31(4):1430-1438, 2013.

\section{DISCUSIÓN}

La formación de células madre depende del nicho en que se encuentra la célula troncal, donde su estado pluripotente o "stemness" esta determinado por la expresión de OCT-4 (Resca et al., 2013). Nuestros resultados describen la expresión de células OCT-4+ en un ubicación anatómica perivascular donde de su nicho se encuentra en la región íntima de la aorta en rata. Un modelo similar del nicho vascular de células madre ha sido previamente descrito con una localización semejante a la de pericitos (Castrechini et al., 2010).

Los resultados confirman el estado inmaduro de las células periciticas al expresar el factor de transcripción Oct4 nuclear que ha sido considerado como el actor principal de los mecanismos de autorenovación y pluripotencia, siendo a su vez, un fundamental represor de las vías moleculares que permiten la diferenciación (Niwa et al., 2000).

Las células aisladas por el método de explante que promueve el aislamiento de células migratorias desde los tejidos muestran un fenotipo con un citoplasma alargado y que expresan aSMA, PDGFRa y $b$, siendo estos dos últimos marcadores específicos de pericitos como ha sido documentado previamente por Bergers \& Song (2005). Ha sido previamente descrito que los pericitos pueden actuar como fuente de células indiferenciadas durante la reparación de tejidos óseo (Diaz-Flores et al., 1992) y adiposo (Crisan et al.; da Silva Meirelles et al.). La distribución anatómica de los pericitos en el cuerpo y su papel en la regeneración ha llevado a plantear que estas células pueden ser una fuente de células mesenquimales (Caplan). Nuestros resultados apoyan esta tesis dado que las células obtenidas expresan Thy1/CD 90 en cultivo, un marcador de células madre mesenquimales. La presencia de la expresión de genes previamente señalados como marcadores de células periciticas PDGFRa, aSMA, PDFGRb que fue evidenciado por RTPCR, se corresponde a lo descrito anteriormente por Bukovsky et al. (2001) y Dore-Duffy \& Cleary.

En cuanto a la expresión de OCT-4, los resultados confirman y amplían aún más las observaciones obtenidas en investigaciones anteriores (Montiel-Eulefi et al., 2009, 2011, 2012), lo que demuestra que las células positivas para OCT-4, se encuentran en los vasos sanguíneos en un microambiente que, probablemente, permite que estas células sobrevivan y/o permanezcan en reposo como un tipo de células madre quiescente (Pasquinelli et al., 2010).

Siguiendo con lo anterior, además de la expresión de OCT-4 es necesario evaluar la ubicación de este factor de transcripción, siendo el núcleo la ubicación descrita para la regulación del estado "stemness" y consistente para señalar al fenotipo celular que lo expresa como célula madre adulta (Landazuri \& Taylor). Gonzalez et al. (2009) han descrito que en células Tipo I de epitelio alveolar de rata Sprague Dawley adulta cultivo expresa OCT4A 52kDa localizado principalmente en el núcleo celular y que lo ha relacionado con la capidad proliferativa y de célula madre de estas células en pulmón. Un resultado similar se observa con la presencia nuclear de OCT-4 en células periciticas en cultivo, y que es diferente para la distribución citoplasmática de OCT-4 de las células en aorta. Esta explicación parece consistente con la diferente expresión génica de OCT-4 de la isoforma $\mathrm{B}$ y de la isoforma A, además por el comportamiento dinámico de OCT-4 aún es desconocido (Oka et al., 2013).

Oka et al. demostraron que OCT-4 es un factor de transcripción que se mantiene translocándose entre el núcleo y el citoplasma, además, la mutación de la región de localización nuclear (NLS) del transportador de OCT-4 produce su difusión pasiva desde el núcleo al citoplasma. Siendo los niveles de OCT-4 en el núcleo fundamentales para mantener un estado indiferenciado de la célula, donde la movilización de este factor dentro y fuera del núcleo es clave más que la expresión de este factor de transcripción. Los resultados en nuestro trabajo muestran en las células perivasculares de aorta y en las células in vitro OCT-4 pueden estar evidenciando una transición desde una forma inactiva a una forma nuclear activa, producto de la translocación del citoplasma al núcleo de OCT-4 y plantean que en el vaso donde OCT-4 se encuentra principalmente citoplasmático estás células se encuentra en un estado quiescente.

\section{ACKNOWLEDGMENTS}

Esta investigación fue financiada por la Universidad de LA Frontera a través del Proyecto PIA DI10-7003 y DI10-7006.

HERRERA-BRAVO, J.; MONTIEL-EULEFI, E.; GLASER, T.; GARCÉS, M; LEAL, P. \& HENNING, U. A. In vitro translocation cytoplasm/nucleus of embryonic transcription factor OCT-4 in perivascular cells suggests that aorta as a niche of quiescent adult stem cells. Int. J. Morphol., 31(4):1430-1438, 2013.

SUMMARY: Perivascular cells have a common origin from embryonic stem cells and blood vessels provide a niche for the maintenance of their stemness. Embryonic markers expression of undifferentiated cells, as well as, the wide variety of cellular 
phenotypes generated from pericytes, could be explained by the ability of these cells to be induced to a state of "stemness" when treated with appropriate factors. Our findings describe the expression of cells with cytoplasmic OCT-4 in perivascular anatomical location where their niche region is in the intima of the aorta in rats. In vitro isolated cells by explant method that promotes the isolation of migratory cells from tissues show an elongated cytoplasm phenotype, expressing aSMA, PDGFRa \& b where the last two are specific markers of pericytes. These cells present a translocated nuclear variant of OCT- 4 that has been described as the master regulator of self-renewal processes and pluripotency. The expression of OCT- 4 further confirms and extends the observations obtained in our previous research and proves that stem cells found in the blood vessels in a microenvironment that probably allows them to survive and remain at rest as a type of quiescent stem cell.

KEY WORDS: Stemness; Pericytes; Translocation; OCT-4; Rat aorta.

\section{REFERENCIAS BIBLIOGRÁFICAS}

Bergers, G. \& Song, S. The role of pericytes in blood-vessel formation and maintenance. Neuro. Oncol., 7(4):452-64, 2005.

Bukovsky, A.; Caudle, M. R.; Keenan, J. A.; Upadhyaya, N. B.; Van Meter, S. E.; Wimalasena, J.; \& Elder, R. F. Association of mesenchymal cells and immunoglobulins with differentiating epithelial cells. BMC Dev. Biol., 1:11, 2001.

Canfield, A. E.; Sutton, A. B.; Hoyland, J. A. \& Schor, A. M. Association of thrombospondin-1 with osteogenic differentiation of retinal pericytes in vitro. J. Cell Sci., 109(Pt. 2):343-53, 1996.

Caplan, A. I. All MSCs are pericytes? Cell Stem Cell, 3(3):22930,2008

Castrechini, N. M.; Murthi, P.; Gude, N. M.; Erwich, J. J.; Gronthos, S.; Zannettino, A.; Brennecke, S. P. \& Kalionis, B. Mesenchymal stem cells in human placental chorionic villi reside in a vascular Niche. Placenta, 31(3):203-12, 2010.

Crisan, M.; Yap, S.; Casteilla, L.; Chen, C. W.; Corselli, M.; Park, T. S.; Andriolo, G.; Sun, B.; Zheng, B.; Zhang, L.; Norotte, C.; Teng, P. N.; Traas, J.; Schugar, R.; Deasy, B. M.; Badylak, S.; Buhring, H. J.; Giacobino, J. P.; Lazzari, L.; Huard, J. \& Péault, B. A perivascular origin for mesenchymal stem cells in multiple human organs. Cell Stem Cell, 3(3):301-13, 2008.

da Silva Meirelles, L.; Caplan, A. I. \& Nardi, N. B. In search of the in vivo identity of mesenchymal stem cells. Stem Cells, 26(9):2287-99, 2008.

Diaz-Flores, L.; Gutierrez, R.; Lopez-Alonso, A.; Gonzalez, R.
\& Varela, H. Pericytes as a supplementary source of osteoblasts in periosteal osteogenesis. Clin. Orthop. Relat. Res., (275):280-6, 1992.

Dore-Duffy, P. \& Cleary, K. Morphology and properties of pericytes. Methods Mol. Biol., 686:49-68, 2011.

Dore-Duffy, P.; Katychev, A.; Wang, X. \& Van Buren, E. CNS microvascular pericytes exhibit multipotential stem cell activity. J. Cereb. Blood Flow Metab., 26(5):613-24, 2006.

Gonzalez, R. F.; Allen, L. \& Dobbs, L. G. Rat alveolar type I cells proliferate, express OCT-4, and exhibit phenotypic plasticity in vitro. Am. J. Physiol. Lung Cell Mol. Physiol., 297(6):L1045-55, 2009.

Howson, K. M.; Aplin, A. C.; Gelati, M.; Alessandri, G.; Parati, E. A. \& Nicosia, R. F. The postnatal rat aorta contains pericyte progenitor cells that form spheroidal colonies in suspension culture. Am. J. Physiol. Cell Physiol., 289(6):C1396-407, 2005 .

Korn, J.; Christ, B. \& Kurz, H. Neuroectodermal origin of brain pericytes and vascular smooth muscle cells. J. Comp. Neurol., 442(1):78-88, 2002.

Landazuri, N. \& Taylor, W. R. The stem cell shell game. Focus on "The postnatal rat aorta contains pericyte progenitor cells that form spheroidal colonies in suspension culture". Am. J. Physiol. Cell Physiol., 289(6):C1361-2, 2005.

Lee, J.; Kim, H. K.; Rho, J. Y.; Han, Y. M. \& Kim, J. The human OCT-4 isoforms differ in their ability to confer self-renewal. J. Biol. Chem., 281(44):33554-65, 2006.

Montiel-Eulefi, E.; Diaz, L. B.; Leal, P.; Roa, J. C.; Risopatron, J.; Salazar, L. A.; Romero, F. \& Sáchez, R. Pericytes: New Approaches in Regenerative Therapy, Cerebrovascular Pathology and Cancer. Int. J. Morphol., 29(3):769-81, 2011.

Montiel-Eulefi, E.; Nery, A. A.; Rodrigues, L. C.; Sanchez, R.; Romero, F. \& Ulrich, H. Neural differentiation of rat aorta pericyte cells. Cytometry A, 81(1):65-71, 2012.

Montiel-Eulefi, E.; Sanchez, R.; Rojas, M. \& Bustos-Obregon, E. Epiblast Embryo Stem Cells Give Origin to Adult Pluripotent Cell Populations: Primordial Germ Cell, Pericytic and Haematopoyetic Stem Cells. A Review. Int. J. Morphol., 27(4):1325-33, 2009.

Niwa, H.; Miyazaki, J. \& Smith, A. G. Quantitative expression of Oct-3/4 defines differentiation, dedifferentiation or selfrenewal of ES cells. Nat. Genet., 24(4):372-6, 2000.

Oka, M.; Moriyama, T.; Asally, M.; Kawakami, K. \& Yoneda, Y. Differential role for transcription factor OCT-4 nucleocytoplasmic dynamics in somatic cell reprogramming and self-renewal of embryonic stem cells. J. Biol. Chem., 288(21):15085-97, 2013. 
HERRERA-BRAVO, J.; MONTIEL-EULEFI, E.; GLASER, T.; GARCÉS, M; LEAL, P. \& HENNING, U. A. La Translocación In Vitro Citoplasma/Núcleo del Factor de Transcripción Embrionario OCT-4 en Células Perivasculares Propone a la Arteria Aorta Como un Nicho Quiescente de Células Madre Adultas. Int. J. Morphol., 31(4):1430-1438, 2013.

Pasquinelli, G.; Pacilli, A.; Alviano, F.; Foroni, L.; Ricci, F.; Valente, S.; Orrico, C.; Lanzoni, G.; Buzzi, M.; Luigi Tazzari, P.; Pagliaro, P.; Stella, A. \& Paolo Bagnara, G. Multidistrict human mesenchymal vascular cells: pluripotency and stemness characteristics. Cytotherapy, 12(3):275-87, 2010.

Pasquinelli, G.; Tazzari, P. L.; Vaselli, C.; Foroni, L.; Buzzi, M.; Storci, G.; Alviano, F.; Ricci, F.; Bonafè, M.; Orrico, C.; Bagnara, G. P.; Stella, A. \& Conte, R. Thoracic aortas from multiorgan donors are suitable for obtaining resident angiogenic mesenchymal stromal cells. Stem Cells, 25(7):162734, 2007.

Ratajczak, M. Z.; Machalinski, B.; Wojakowski, W.; Ratajczak, J. \& Kucia, M. A hypothesis for an embryonic origin of pluripotent Oct-4(+) stem cells in adult bone marrow and other tissues. Leukemia, 21(5):860-7, 2007.

Reilly, T. M.; Seldes, R.; Luchetti, W. \& Brighton, C. T. Similarities in the phenotypic expression of pericytes and bone cells. Clin. Orthop. Relat. Res., (364):95-103, 1998.

Resca, E.; Zavatti, M.; Bertoni, L.; Maraldi, T.; De Biasi, S.; Pisciotta, A.; Nicoli, A.; La Sala, G. B.; Guillot, P. V.; David, A. L.; Sebire, N. J.; De Coppi, P. \& De Pol, A. Enrichment in c-Kit(+) enhances mesodermal and neural differentiation of human chorionic placental cells. Placenta, 34(7):526-35, 2013.

Tárnok, A.; Ulrich, H. \& Bocsi, J. Phenotypes of stem cells from diverse origin. Cytometry A, 77(1):6-10, 2010.

Terskikh, A. V.; Easterday, M. C.; Li, L.; Hood, L.; Kornblum, H. I.; Geschwind, D. H. \& Weissman, I. L. From hematopoiesis to neuropoiesis: evidence of overlapping genetic programs. Proc. Natl. Acad. Sci. U S A, 98(14):7934-9, 2001.

Yamanaka, S. \& Takahashi, K. Induction of pluripotent stem cells from mouse fibroblast cultures. Tanpakushitsu Kakusan Koso, 51:2346-51, 2006.

Zengin, E.; Chalajour, F.; Gehling, U. M.; Ito, W. D.; Treede, H.; Lauke, H.; Weil, J.; Reichenspurner, H.; Kilic, N. \& Ergün, S. Vascular wall resident progenitor cells: a source for postnatal vasculogenesis. Development, 133(8):1543-51, 2006.

\author{
Dirección para correspondencia: \\ Enrique Montiel-Eulefi \\ Centro de Biotecnología en Reproducción \\ (CEBIOR-CEGIN-BIOREN) \\ Departamento de Ciencias Básicas \\ Facultad de Medicina \\ Universidad de La Frontera \\ Montevideo 0870, Temuco \\ CHILE
}

Email: emontiele@gmail.com

Recibido: 24-07-2013

Aceptado: 07-11-2013 